\title{
Quantum Well Interference and Exchange Coupling in Double Quantum Well Thin Films
}

\author{
Zhang Zhi-dong \\ Shenyang National Laboratory for Materials Science and International Centre for \\ Materials Physics, Institute of Metal Research, Academia Sinica, Wenhua Road 72, \\ Shenyang 110016, Peoples' Republic of China
}

A comprehensive review is given of recent advances in understanding quantum well interference $(\mathrm{QWI})$ in double quantum wells $(\mathrm{DQW})$. The quantum well interference in double quantum wells is represented, based on the one - electron effective mass model. The probability of the electrons to be found in the several double quantum wells is derived analytically, providing with more detailed information on QWI. The calculation of the probability of the electron in the wells, based on effective mass theory, is in consistent with the density of states in metal thin films revealed by photoemission at the Fermi edge, which were performed at the third generation Advanced Light Source (ALS) at the Lawrence Berkeley National Laboratory. The beamline 7.0.1.2 at the ALS can focus the photon beam down to a 50-100 $\mu \mathrm{m}$ spot size with a photon flux (>10 12 photons per second at resolving power of 10000) to do the photoemission experiments. Thus, for a wedge of $\sim 10 \mathrm{ML} / \mathrm{mm}$, a scan of a $50 \mu \mathrm{m}$ photon beam across the sample provides a systematic thickness-dependent measurement with $\sim 0.5 \mathrm{ML}$ thickness resolution.

According to our calculations, the exchange coupling is found to correlate directly to the quantum interference. The quantum well interference in the double quantum well depends sensitively on the relative magnitudes of the wavevectors of metals as well as the amplitudes of the density of states induced by different wavevectors. The phase accumulation method is still appropriate for interpreting the QWI in DQW systems. The symmetry of the quantum wells on quantization condition, and the special feature of the density of states are discussed. The phase accumulation relation could be linear for each well, but non-linear for the whole system in the 
symmetric DWQ. In the asymmetric DQW system, the linear quantization condition is valid only for part of layers whereas the quantization condition for others may be non-linear due to the quantum well interference. The non-linear quantization condition is a character of the DQW, which distinguishes with that of the SQW.

Neglecting the spin - dependent effects, we have illustrated successfully the oscillation of the probability of the electrons in the DQW systems. The oscillation periodicity of the probability due to the QWI is in good agreement with the experimental data for that of the exchange coupling and the giant magnetoresistance. We argue that the pure quantum interference is a possible mechanism for the oscillation of the exchange coupling and the giant magnetoresistance, besides the spin-dependent scattering. Because this mechanism is only the response of the electrons at the Fermi surface, the probability of electrons in the double quantum wells constructed all by ferromagnetic layers oscillates as the thickness of the spacer layer increases. Thus the oscillation of the exchange coupling between ferromagnetic layers separated by ferromagnetic layer is predicted.

All the results suggest that each layer in an entire multilayer stack is relevant to the magnetic coupling, thus favoring the QW coupling, in good agreement with the photoemission experiments. This work strongly supports the QW picture of the magnetic coupling, confirming that the oscillations of the interlayer exchange coupling correspond to the effective Fermi vectors $\mathrm{k}^{\text {eff }}$ of the layers.

The advantage and the limitation of the present model are discussed, in comparison with those of the first principles approach. The present work suggests that the effective mass theory could be used to fit and to interpret the experimental data of the multi-layers, even in the mono-layer limit. This model is an easy to use and rather effective tool for such systems. In some cases, this simple model could be used to predict new phenomena in the ultrathin films.

This work has been supported by the National Natural Sciences Foundation of China under Grant No. 59725103. The author thanks Prof. Dr. Z. Q. Qiu of the Department of Physics, University of California at Berkeley for helpful discussions. 